\title{
Potential drug targets for neuroregeneration and repair
}

\begin{abstract}
Unlike the peripheral nervous system (PNS) the adult mammalian central nervous system (CNS) does not spontaneously regenerate after injury. Numerous axon-inhibitory molecules are present in the injured CNS and various strategies for overcoming these obstacles and enhancing CNS regeneration had been experimentally developed. Adult neuroregeneration is a complex process, beyond the common knowledge of neurogenesis that also comprises endogenous neuroprotection leading to neuroplasticity and neurorestoration, a therapeutical approach of implantation of viable cells. Regeneration in the CNS implies that new neuron generate either through proliferation of endogenous stem/progenitor cells or by administration of exogenous stem/precursor cells with potential to substitute for lost tissue, or can be maintained by targeting the axon inhibitory molecule in the CNS. Here, the main focus of review emphasis on the axon inhibitory molecules, which prevents neuroregeneration and repair.
\end{abstract}

Volume 4 Issue 4 - 2017

\author{
Ajay Kumar, Nandan Singh, Alka Kurmi, \\ Shashi Bhushan Tripathi, Savita Chauhan, \\ Abhishek Singh, Akash Pratap Singh, \\ Dharmendra Saikia \\ Department of Molecular Bioprospection, India
}

\author{
Correspondence: Dharmendra Saikia, CSIR- Central Institute \\ of Medicinal and Aromatic Plants, Lucknow 226 015, Uttar \\ Pradesh, India, Tel 0522-27I8650, \\ Email dsaikia.cimap@gmail.com
}

Received: March 22, 2017 | Published: April 07, 2017

Keywords: neuroregeneration, proteoglycans, nogo, sema-4d, mesenchymal stem cells, carbon nanotubes

Abbreviations: PNS, peripheral nervous system; CNS, central nervous system; MAG, myelin associate glycoprotein; CSPGs, chondroitin sulphate proteoglycans; ECM, extracellular matrix; HSPGs, Heparin Sulphate Proteoglycans; GFAP, glial fibrillary acidic protein; KSPGs, keratan sulphate proteoglycans; GlcNAc, galactose and $N$-acetylglucosamine

\section{Introduction}

Neuroregeneration refers to the repair or re-growth of nervous tissues by generation of new neurons, axons, glial cells, myelin, or synapses. ${ }^{1}$ Since perpetuity, damage to the CNS was untreatable. No effective treatment regimen was available for rejuvenate nerve function after injury to the CNS, and multiple attempts at neural regrowth were unsuccessful ${ }^{2}$ simply due to a lack of knowledge of CNS regeneration. In recent past, the discovery that adult CNS neurons are able to reproduce after injury has overcome this therapeutic nihilism. Nervous system injuries affected the lives of thousands of people every year. Due to high incidence of neurological injuries, the field of neuroregeneration research has been advancing rapidly over the past few years and has provided attractive new insights into the physiological functions and pathogenic roles of a broad range of molecules linked with several annihilating neurodegenerative disorders, including Alzheimer's, Parkinson's, Amyotrophic lateral sclerosis, Down syndrome, Fronto-temporal dementia and Huntington's disease.

Nervous system is divided into two major parts viz. CNS, which consists of the brain and spinal cord, and the PNS, which consists of cranial and spinal nerves along with the associated ganglia. Previous studies reveals that neuroregeneration in the PNS is not uncommon. Damage of neurons in the PNS immediately induces the migration of macrophages and phagocytes to the lesion site. Axonal sprouts then form at the proximal stump and grow until they enter the distal stump. Previous finding explains that axons are able to re-grow as long as the cell body is intact. Unlike PNS injury, CNS injury is not following the extensive regeneration. ${ }^{3}$ Regeneration in the CNS is limited due to inhibitory influences of glial cells and the complex, impermissible extracellular growth environment, which is formed by the migration of myelin-associated inhibitors, astrocytes, microglia, oligodendrocytes and their forerunner. The environment in the CNS, especially after trauma and invasive damage, prevents the repair of myelin and neurons. ${ }^{4}$ In addition to myelin, glial scar tissue that forms at the lesion site also suppresses fiber growth. Inhibitory myelin and axonal debris not cleared rapidly after CNS injury, which may contribute to glial scar formation. The proximal axons attempt to regenerate after injury; however, their growth is affected by the glial scar formation. It is important to note that the primary problem with axonal regeneration in the CNS is to waive the inhibitory environment associated with axonal regeneration. ${ }^{5}$

\section{Inhibition of axonal regrowth}

Earlier studies have reported that glial scar formation may significantly inhibit nerve regeneration following damage to the CNS. ${ }^{6}$ In addition, the adverse environment is a major inhibitor of fruitful recovery after injury to the CNS. Molecules, such as transforming growth factors beta-1( $\beta-1)$ and beta-2( $\beta-2)$, cytokines, interleukins, chondroitin sulphate proteoglycans, phosphacan, and neurocan believed to promote glial scar formation (gliosis). Further, astrocytes may aid in the upregulation of chondroitin sulphate proteoglycans, thereby inhibiting nerve regeneration. These upregulated molecules may affect the composition of the extracellular matrix and inhibit neurite outgrowth in the CNS. Additionally, molecules such as chondroitin sulphate proteoglycans, ephrins, EphA4, and Sema3A provoke inhibition of axonal regeneration. Previous studies revealed that the expression of phosphacan significantly declined in glial scar tissue in comparison to normal brain tissue. In addition, neurocan level was elevated in astrocytes in glial scars and it remains elevated one month after the initial injury. Nogo, a protein family has been associated with the inhibition of re-myelination in the CNS. Studies suggested that Nogo A may play a key role in autoimmune-mediated de-myelination. Nogo-66 is responsible for neurite outgrowth inhibition. Nogo A functions via its amino-Nogo terminus or by its Nogo-66 terminus. 
Oligodendrocyte myelin glycoprotein and myelin associate glycoprotein(MAG) may activate receptor $\mathrm{NgR}$ a protein that in humans encoded by RTN4G gene mediates plasticity and regulates axonal regeneration. Additionally, lipopolysaccharide or lipooligosaccharide may impart a protective mechanism in neuroregeneration. Recently, Bingham et al. reported lipopolysaccharide treatment after injury protects rat neuronal and glial cell cultures. ${ }^{7}$

\section{Chondroitin Sulphate Proteoglycans}

Chondroitin sulphate proteoglycans (CSPGs) are extracellular matrix (ECM) component that are generally expressed in cartilage and in the developing and adult CNS, these molecules impart a key characteristics in neuronal development and glial scar formation. They are also involved in cell processes, such as adhesion, growth, receptor binding, migration, and interaction of cell with the other extracellular matrix constituents. They are also certain to acts between, among with laminin, fibronectin, tenascin, and collagen protein in ECM. ${ }^{8}$ CSPGs generally secreted from cells. They are of two types, chondroitin sulphate (CSPGs) and heparin sulphate (HSPGs). The CSPGs acts as a barrier-forming molecule, whereas the HSPGs stabilise the communication of receptors and ligands. During developmental stage CSPGs patterns the cell migration, axon growth pathways and axon terminations. Later in adulthood, CSPGs associate with some classes of neuron and control plasticity. After damage to the CNS, CSPGs are the major axon growth inhibitory, smallest unit of the glial scar tissue that keeps from doing good regeneration. CSPGs have a diverse role in the nervous system, including binding to the molecules and blocking their action, presenting molecules to cells and axons, localising active molecules to particular sites and presenting growth factors to their receptors. In vitro studies demonstrate their potential to restrict neurite outgrowth and it is believed to inhibit axonal regeneration after CNS injury in vivo. Previous studies indicate that CSPGs are generally upregulated after spinal cord injury, and more recent research have begun to identify individual proteoglycans that may play dominant roles in limiting axonal regeneration. The study examined the extended deposition patterns after CNS injury of four putatively inhibitory CSPGs that has not been extensively investigated previously in vivo: neurocan, brevican, phosphacan, and versican. After injury to spinal cord, neurocan, brevican, and versican immunolabeling raised within days in injured spinal cord parenchyma surrounding the lesion site and spike at 2 weeks. Neurocan and versican were persistently elevated for 4 weeks postinjury, and brevican expression persisted for at least 8 weeks. On the other hand, phosphacan immunolabeling decreased in the same region immediately following injury but later recovered and then peaked after 8 weeks. Combined glial fibrillary acidic protein (GFAP) immunohistochemistry and in situ hybridization demonstrated that GFAP astrocytes constituted a source of neurocan production after spinal cord injury. Thus, the production of several CSPGs family members is affected by spinal cord injury, overall establishing a CSPGs-rich matrix that persists for up to 8 weeks following injury. Optimization of strategies to reduce CSPGs expression to enhance regeneration may need to target several different family members over an extended period following injury. ${ }^{8-10}$

\section{Keratan Sulphate Proteoglycans}

Keratan sulphate proteoglycans (KSPGs) augmented after CNS injury and act as inhibitory cues. KSPGs induced in the lesion of CNS injury and regarded as nonpermissive cue to neuronal axon regeneration. ${ }^{11,12}$ However, roles of KSPGs in CNS injury have not studied extensively. KSPGs a glycosaminoglycan, which is elongated from $N$ - or $O$-glycans covalently, attached to scaffold proteins. KSPGs composed of repeating disaccharide units of galactose and $\mathrm{N}$-acetylglucosamine (GlcNAc), with sulphate residues at the C6 position of galactose and GlcNAc. As GlcNAc sulfation at the C6 position is necessary for KSPGs chain elongation ${ }^{13}$ failure in this sulfation may lead to loss of KSPGs synthesis. Previous studies reported that KSPGs expression in the developing brain is detectable with 5D4, a KSPGs -specific monoclonal antibody. These 5D4 immunoreactivities can eliminate in mice deficient in $N$-acetylglucosamine 6-O-sulfotransferase-1. In mice, brain injury upregulates mRNA expression of $\mathrm{N}$-acetylglucosamine $6-O$-sulfotransferase- 1 as well as 5D4-reactive KSPGs in the wounded area. Intriguingly, the expression of 5D4-reactive KSPGs and reactive astrocyte accumulation in the wounded area found decreased in the sulfotransferase-deficient mice. Consequently, the deficient mice exhibit a marked reduction in scar formation and enhancement of neuronal regeneration after brain injury. Thus, $\mathrm{N}$-acetylglucosamine 6 - $O$-sulfotransferase-1 plays indispensable roles in brain KSPGs biosynthesis and glial scar formation after brain injury. ${ }^{14,15}$

\section{Growth-Inhibitory Molecules in the CNS}

A variety of molecule's known to exist in the adult CNS have been shown through in vitro and in vivo studies to demonstrate axonal growth-inhibitory properties. More importantly, many of these molecules are specific to, or upregulated in the injured CNS environment. These molecules classified into two main types: myelinassociated molecules and Extracellular matrix constituents.

\section{Myelin Associated Molecules}

In the late 1980s Schwab and colleagues demonstrated that a substrate non-permissive to growth exists in CNS white matter ${ }^{16-19}$ leading to the hypothesis that where myelinated axons are disrupted, debris containing myelin-associated axon-inhibitory molecules will be present around the lesion.

A number of myelin-associated molecules have since been isolated that have axon-inhibitory properties, including the two neurite growth inhibitors Nogo (originally called NI-250) and myelin-associated glycoprotein (MAG).

\section{Extracellular Matrix Constituents (ECM) Constituents}

For many neurons, their migration and axon elongation occurs through the ECM. In the CNS the ECM is largely devoid of cells but contains several types of molecules with which neurons and glia interact, and these can have important influences on many aspects of a cell's behaviour. The extracellular space surrounding many nonneuronal cells in the CNS is filled with a network of glycoproteins, proteoglycans and hyaluronan, close to the membrane of such cells the ECM becomes more dense, forming a basement membrane composed principally of collagens; glycoproteins - particularly tenascin- C and tenascin-R; chondroitin sulphate proteoglycans and heparan sulphate proteoglycans; hyaluronic acid; cell adhesion molecules and integrins. ECM molecules expressed in the developing CNS may have both growth-promoting and growth-inhibitory effects on axons. ${ }^{20-25}$ Many of these molecules are upregulated in the adult ECM following a lesion to the CNS and have been shown to have inhibitory properties towards regenerating axons. Tenascin is an important secreted ECM component with a range of binding sites and functions. ${ }^{26-30}$ Tenascin is abundant in the basement membrane, being produced by astrocytes 
during development, with important roles in mediating axon-glia interactions. ${ }^{27,30,31}$ There are two members of the tenascin gene family: tenascin- $\mathrm{C}$ and tenascin- $\mathrm{R}$; tenascin- $\mathrm{C}$ is expressed as numerous alternatively spliced variants with various functions. ${ }^{32-34}$ The same tenascin molecule may have either growth-inhibitory or growth promoting effects towards different neurons within different contexts; a number of studies have demonstrated the neurite growth-inhibitory properties of tenascin in vitro. ${ }^{35,36}$ It also has growth-promoting effects ascribed to the alternatively spliced A-D and D5 domains. ${ }^{37,38}$ Tenascin is found in the normal adult CNS although at lower levels than in the developing CNS. Production is up-regulated in the glial scar after injury the increased tenascin has been co-localized with reactive glial fibrillary acidic protein (GFAP) and astrocytes. ${ }^{39}$ Tenascin is known to interact with many CSPGs in vitro ${ }^{28}$ and thus may be capable of forming (axon inhibitory) complexes with CSPGs in injured CNS tissue.

\section{Other Inhibitory Factors}

\section{Nogo}

Nogo, also known as Reticulon-4, is a protein, in humans is encoded by the RTN4 gene ${ }^{40,41}$ that is specific to the central nervous system (CNS), and has been identified as an inhibitor of neurite outgrowth. Reticulons are associated with the endoplasmic reticulum, and are involved in neuroendocrine secretion or in membrane trafficking in neuroendocrine cells. Nogo-A is the largest member of the Nogo family and is responsible for inhibition of CNS regeneration. There are three isoforms: Nogo A, B and C. Nogo-A has two known inhibitory domains including amino-Nogo, at the N-terminus and Nogo-66, which makes up the molecules extracellular loop. Both amino-Nogo and Nogo-66 are involved in inhibitory responses, where amino-Nogo is a strong inhibitor of neurite outgrowth, and Nogo-66 is involved in growth cone destruction. The Nogo-66 receptor (NgR), a membrane protein which binds to Nogo, may play an important role in signal transduction for several myelin-associated inhibitors. The discovery of the Nogo family and the NgR provides an opportunity to develop interventions to promote axonal regeneration in the CNS after brain injury. Basic and clinical research of Nogo has increased our understanding of the mechanisms underlying spinal cord injury, multiple sclerosis, and neurodegenerative diseases. Research suggests that blocking Nogo-A during neuronal damage will help to protect or restore the damaged neurons. ${ }^{42}$ The investigation into the mechanisms of this protein presents a great potential for the treatment of autoimmune mediated demyelinating diseases and spinal cord injury regeneration. It has also been found to be a key player in the process whereby physical exercise enhances learning and memory processes in the brain. Understanding the biological functions of Nogo family members may open up a new avenue for the development of therapeutic agents.

\section{Myelin-associated glycoprotein and its axonal receptors}

Myelin-associated glycoprotein (MAG) is a cell surface member of the immunoglobulin-like (Ig) superfamily, with five extracellular Ig-like domains, a single transmembrane domain, and one of two alternatively spliced short cytoplasmic tails. ${ }^{43}$ It is coded by a single gene that is conserved among vertebrates, ${ }^{44}$ human and rodent MAG are $95 \%$ identical at the amino acid level over the entire extracellular expressed domain (5 Ig-like domains, 500 amino acids). MAG is produced only in myelinating glial cells: oligodendrocytes in the CNS and Schwann cells in the PNS, although it is a quantitatively minor myelin protein (comprising 1\% of CNS and $0.1 \%$ of PNS myelin protein) MAG is not expressed uniformly throughout myelin. In the CNS, MAG is located on the inner-most (periaxonal) non-compacted myelin wrap. ${ }^{45}$ In the PNS MAG is found on periaxonal myelin, and on other noncompacted myelin (paranodal loops, Schmitt-Lanterman incisures, and the mesaxon). Myelination of axons provides for rapid nerve conduction that is essential to vertebrate nervous system function. In addition to providing segmental insulation, myelin enhances axon survival, regulates the axon cytoskeleton, directs the distribution of molecules at nodes of Ranvier, and inhibits axon regeneration after injury ${ }^{46-48}$ Knowledge of the myelin molecules and axon receptors responsible for the nurturing and inhibiting effects of myelin on axons may provide insight into the basis of dysmyelinating disorders and provide lead molecules to enhance axon regeneration after injury or disease.

\section{Sema-4D}

Semaphorin 4D (Sema 4D) is an axon guiding molecule which is secreted by oligodendrocytes and induces growth cone collapse in the central nervous system. Sema4D, also called CD100, was first isolated in the immune system where it is involved in $\mathrm{B}$ and $\mathrm{T}$ cell activation. Sema4D is expressed in cells throughout the CNS white matter, with a peak during the myelination period. There are more than 20 known semaphorins grouped into eight classes: classes 1 and 2 are invertebrate semaphorins, classes 3 to 7 are found in vertebrates, and class 5 and 8 , has been identified in some non-neurotropic DNA viruses. Sema4D is composed of a Sema domain, a Cystine Rich domain also called the Plexin Repeat Domain or the Met Related Sequence. The Cystine Rich domain has an unknown function but is found in several different receptors. Three copies of this repeat are found in Plexin-B1, the receptor for Sema 4D, ${ }^{49}$ while the Met receptor contains one copy. Immunoglobulin family members include components of immunoglobulins and cell surface glycoproteins such as the T-cell receptors CD2, CD4, and CD8. The function of the Sema4D intracellular domain is not known, but it has been associated with a serine kinase activity, suggesting bi-directional signaling may take place. By binding with plexin $\mathrm{B} 1$ receptor it functions as an R-Ras GTPase-activating protein (GAP) and repels axon growth cones in both the mature central nervous system..$^{50,51}$ In the immune system, CD100 binds CD72 to activate B cells and dendritic cells, though much about this interaction is still under investigation..$^{52,53}$

During skin damage repairs, SEMA4D interacts with Plexin B2 on gamma delta $t$ cells to play a role in the healing process. ${ }^{54}$ Soluble forms of Sema4D had neurotrophic effects which were inhibited by neutralizing antibodies to Sema4D. Sema4D strikingly potentiated neurite outgrowth in the presence of $50 \mathrm{ng} / \mathrm{ml} \mathrm{NGF}$ and increased sensitivity to NGF. Cells responded to very low concentrations of NGF in the presence of 1nM Sema4D. Activation of following signal proteins, protein kinase $\mathrm{C}$ (PKC), L-type of voltage-dependent $\mathrm{Ca} 2+$ channel, and phosphatidylinositol (PI) 3-kinase mediated neurotrophic neurite-outgrowth action of Sema4D. These findings suggest a new function of Sema4D as a neurotrophic signal in PC12 cells. ${ }^{55}$

\section{Treatment Strategies for Neuroregeneration}

\section{Living scaffolds for neuroregeneration}

Neural tissue engineers are working out on key mechanisms important for axonal pathfinding and neural cell moving during early embryonic development to make come into existence living scaffolds for neuroregeneration following damage and disease. Mechanistic approach involve the combined use of haptotactic, chemotactic, and 
mechanical cues to explicit cell movement and re-growth. Living scaffolds provide these cues through the use of cells engineered in a predefined architecture, generally in combination with biomaterial strategies. Although several hurdles exist in the implementation of living regenerative scaffolds, there are considerable therapeutic advantages to using living cells in conjunction with biomaterials. The leading contemporary living scaffolds for neuro repair are utilizing aligned glial cells and neuronal/axonal tracts to direct regenerating axons across damaged tissue to appropriate targets, and in some cases to directly replace the function of lost cells. Future advances in technology, including the use of exogenous stimulation and genetically engineered stem cells, will further the potential of living scaffolds and drive a new era of personalized medicine for neuroregeneration. ${ }^{56}$

\section{Mesenchymal stem cells}

Mesenchymal stem cells (MSC) initially termed mesenchymal stromal cells, were first identified by Friedenstein and co-workers. Stem cell-based therapies emerge as a possible strategy to treat diseases of the CNS.${ }^{57} \mathrm{MSC}$ are of particular interest for regenerative therapies not only due to their multipotentiality, but also mainly because these cells have proregenerative and immunomodulatory properties The bone marrow-derived MSC is the most studied type of MSC. They have potential to differentiate into adipocytes, chondroblasts and osteoblasts. ${ }^{58}$ Several authors have described differentiation into myocytes $^{59}$ and to some extent into neuronal and glial cells. Over 10 years ago, it was claimed that MSC are not only able to differentiate into the cells of mesenchymal lineages but also into neurons ${ }^{60}$ and glial cells ${ }^{61}$ based on expression of neuronal and glial markers in vitro. In the subsequent years, several reports have confirmed those observations. ${ }^{62}$ Earlier studies claims MSC exert neurotrophic effects by releasing a complement of molecules that directly or indirectly promote endogenous repair. Such molecules may include neurotrophic growth factors, chemokines, cytokines, and extracellular matrix proteins. The effect of such factors can be very broadly classified as angiogenic, neurogenic, neuroprotective, synaptogenic and inhibition of scarring. ${ }^{63}$ Paracrine effects involve direct neurotrophic and/or neuroprotective activity on either resident progenitor cells, hence inducing neurogenesis/oligodendrogenesis, or protective, antiapoptotic effects on neurons or glia cells, neurite outgrowth and angiogenesis. Potent anti-inflammatory and immunoregulatory effects represent unique features of MSC, among all stem cell types. Most of the data related to these effects has been obtained in vitro; however, it is conceivable that MSC may have similar regulatory function in vivo, either in their perivascular location or after grafting. ${ }^{64}$

\section{Carbon nanotubes}

A decade of advances in nanotechnology has disclosed exciting perspectives and innovative approaches for tissue regeneration and, more recently, for nerve tissue repair and tune nerve cell behaviour. ${ }^{65,66}$ In this scenario, carbon nanotubes are placed as promising players. Such materials are at the leading edge of nanotechnology, due to carbon nanotube well documented electrical, thermal and mechanical properties. ${ }^{67}$ Carbon nanotube cylindrical morphology is reminiscent of that of distal neuronal dendrites ${ }^{68}$ small cellular processes crucially involved in the ability of neurons to express complex computational skills. This similarity, together with carbon nanotube topographic features, physical properties, as conductivity, and surface-to-volume ratio, ${ }^{69}$ sets the stage for carbon nanotube exploitation in devices able to interface neuronal physiology. The use of nanomaterials in the design of tissue scaffolds in the CNS is primarily due to their abilities to favour neuronal adhesion, to re-create an ECM-like microenvironment and to interact with neuronal membranes at the nanoscale. In fact, a fundamental step, in any strategies aimed at improving CNS regenerative ability, is the manufacturing of scaffolds which are able to control and tune cellular adhesion, ${ }^{70}$ to govern axonal regrowth and neuronal physiology. ${ }^{71,72}$ The achievements in chemically functionalizing carbon nanotubes pushed the development of a variety of soluble forms of nanotubes for molecular sensing, diagnostics, drug delivery and for use as contrast agents. From the neuronal perspective, soluble functionalized carbon nanotubes can be internalized by neurons and usually affect neuronal performance. The first evidence of an impact of soluble carbon nanotubes on neuronal morphology came in 2005 , when $\mathrm{Ni}$ and colleagues $^{73}$ demonstrated that water-soluble carbon nanotubes functionalized with PABS or PEG applied to dissociated hippocampal neurons in vitro induce an increase in neurite length, paralleled by a reduction in the number of neurites and growth cones. Calcium imaging experiments allowed the same authors to show that SWCNT-PEG inhibited the cell depolarization-dependent calcium influx and such an effect possibly explains their impact on morphology. In line with these results, soluble carbon nanotubes functionalized with PEG were also reported to induce a block of endocytosis,$^{74}$ step forward in the exploitation of soluble carbon nanotubes for neuroregenerative purposes has been recently made by the work of Roman and colleagues. ${ }^{75}$

\section{Acknowledgements}

None.

\section{Conflicts of Interest}

The authors do not have any personal or financial interests.

\section{Funding}

None.

\section{References}

1. Zheng B, Ho C, Li S, Keirstead H, et al. Lack of enhanced spinal regeneration in Nogo-deficient mice, Neuron. 2003;38(2):213-224.

2. Kim JE, Li S, Grand Pre T, et al. Axon regeneration in young adult mice lacking Nogo-A/B. Neuron. 2003;38(2):187-199.

3. Faulkner JR, Herrmann JE, Woo MJ, et al. Reactive astrocytes protect tissue and preserve function after spinal cord injury. $J$ Neurosci. 2004;24(9): 2143-2155.

4. McClellan AD. Special issue on spinal cord injury in brain research bulletin. Brain Res. Bull. 2011;84(4-5):265-266.

5. Domeniconi M, Cao Z, Spencer T, et al. Myelin-associated glycoprotein interacts with the Nogo66 receptor to inhibit neurite outgrowth. Neuron. 2002;35(2):283-290.

6. Wong ST, Henley JR, Kanning KC, et al. A p75(NTR) and Nogo receptor complex mediates repulsive signaling by myelin associated glycoprotein. Nat Neurosci. 2002;5(12):1302-1308.

7. Bingham D, John CM, Panter SS, et al. Post-injury treatment with lipopolysaccharide or lipooligosaccharide protects rat neuronal and glial cell cultures. Brain Res Bull. 2011;85(6):403-409.

8. Rhodes KE, Fawcett JW. Chondroitin sulphate proteoglycans: preventing plasticity or protecting the CNS? J Anat. 2004;204(1):33-48.

9. Jones LL, Margolis RU, Tuszynski MH. The chondroitin sulphate proteoglycans neurocan, brevican, phosphacan, and versican are differentially regulated following spinal cord injury. Exp Neurol. 2003;182(2):399-411. 
10. Carulli D, Laabs T, Geller HM, et al. Chondroitin sulphate proteoglycans in neural development and regeneration. Curr Opin Neurobiol. 2005;15(1):116-120.

11. Silver J, Miller JH. Regeneration beyond the glial scar. Nat Rev Neurosci. 2004;5(2):146-151.

12. Jones LL, Tuszynski MH. Spinal cord injury elicits expression of keratan sulphate proteoglycans by macrophages, reactive microglia, and oligodendrocyte progenitors. J Neurosci. 2002;22(11):4611-4624.

13. Akama TO, Nakayama J, Nishida K. Human corneal GlcNac 6Osulfotransferase and mouse intestinal GlcNac 6-O-sulfotransferase both produce keratan sulphate. J Biol Chem. 2001;276(19):1627116278 .

14. Akama TO, Nishida K, Nakayama J, et al. Macular corneal dystrophy type I and type II are caused by distinct mutations in a new sulphotransferase gene. Nat Genet. 2000;26(2):237-241.

15. Zhang HQ, Uchimura K, Kadomatsu K. Brain keratan sulphate and glial scar formation. Ann NY Acad Sci. 2006;1086: 81-90.

16. Schwab ME, Thoenen H. Dissociated neurons regenerate into sciatic but not optic nerve explants in culture irrespective of neurotrophic factors. $J$ Neurosci. 1985;5(9):2415-2423.

17. Schwab ME, Caroni P. Oligodendrocytes and CNS myelin are nonpermissive substrates for neurite growth and fibroblast spreading in vitro. J Neurosci. 1988;8(7):2381-2393.

18. Crutcher KA. Tissue sections from the mature rat brain and spinal cord as substrates for neurite outgrowth in vitro: extensive growth on gray matter but little growth on white matter. Exp Neurol. 1988;104(1):39-54.

19. Savio T, Schwab ME. Rat CNS white matter, but not gray matter, is nonpermissive for neuronal cell adhesion and fiber outgrowth. $J$ Neurosci. 1989;9(4):1126-1133.

20. Snow DM, Steindler DA, Silver J. Molecular and cellular characterization of the glial roof plate of the spinal cord and optic tectum: a possible role for a proteoglycan in the development of an axon barrier. Dev Biol. 1990;138(2):359-376.

21. Oakley RA, Tosney KW. Peanut agglutinin and chondroitin-6-sulphate are molecular markers for tissues that act as barriers to axon advance in the avian embryo. Dev Biol. 1991;147(1):187-206.

22. Brittis PA, Canning DR, Silver J. Chondroitin sulphate as a regulator of neuronal patterning in the retina. Science. 1992;255(5045):733-736.

23. Emerling DE, Lander AD. Inhibitors and promoters of thalamic neuron adhesion and outgrowth in embryonic neocortex: functional association with chondroitin sulphate. Neuron. 1996;17(6):1089-1100.

24. Treloar HB, Nurcombe V, Key B. Expression of extracellular matrix molecules in the embryonic rat olfactory pathway. $J$ Neurobiol. 1996;31(1):41-55.

25. Margolis RU, Margolis RK. Chondroitin sulphate proteoglycans as mediators of axon growth and pathfinding. Cell Tissue Res. 1997;290(2):343-348

26. Hoffman S, Crossin KL, Edelman GM. Molecular forms, binding functions, and developmental expression patterns of cytotactin and cytotactin-binding proteoglycan, an interactive pair of extracellular matrix molecules. J Cell Biol. 1988;106(2):519-532.

27. Steindler DA, Cooper NG, Faissner A, et al. Boundaries defined by adhesion molecules during development of the cerebral cortex: the J1/ tenascin glycoprotein in the mouse somatosensory cortical barrel field. Dev Biol. 1989;131(1):243-260.

28. Grumet M, Milev P, Sakurai T, Karthikeyan L, et al. Interactions with tenascin and differential effects on cell adhesion of neurocan and phosphacan, two major chondroitin sulphate proteoglycans of nervous tissue. J Biol Chem. 1994;269(16):12142-12146.
29. Husmann K, Carbonetto S, Schachner M. Distinct sites on tenascin-C mediate repellent or adhesive interactions with different neuronal cell types. Cell Adhes Commun. 1995;3(4): 293-310.

30. Faissner A, Kruse J. J1/tenascin is a repulsive substrate for central nervous system neurons. Neuron. 1990;5(5): 627-637.

31. Lochter A, Vaughan L, Kaplony A, et al. J1/tenascin in substrate-bound and soluble form displays contrary effects on neurite outgrowth. $J$ Cell Biol. 1991;113(5):1159-1171.

32. Faissner A, Kruse J, Chiquet-Ehrismann R, et al. The high-molecularweight J1 glycoproteins are immunochemically related to tenascin. Differentiation. 1988;37(2): 104-114.

33. Stern CD, Norris WE, Bronner-Fraser M, et al. J1/tenascin related molecules are not responsible for the segmented pattern of neural crest cells or motor axons in the chick embryo. Development. 1989;107(2):309-319.

34. Chuong CM, Chen HM. Enhanced expression of neural cell adhesion molecules and tenascin (cytotactin) during wound healing. Am J Pathol. 1991;138(2):427-440.

35. Pesheva P, Spiess E, Schachner M. J1-160 and J1-180 are oligodendrocyte-secreted nonpermissive substrates for cell adhesion. $J$ Cell Biol. 1989;109(4):1765-1778.

36. Crossin KL, Prieto AL, Hoffman S, et al. Expression of adhesion molecules and the establishment of boundaries during embryonic and neural development. Exp Neurol. 1990;109(1):6-18.

37. Meiners S, Mercado ML, Nur-e, - Kamal MS, et al. Tenascin-C contains domains that independently regulate neurite outgrowth and neurite guidance. J Neurosci. 1999;19(19):8443-8453.

38. Meiners S, Nure-Kamal MS, Mercado ML. Identification of a neurite outgrowth-promoting motif within the alternatively spliced region of human tenascin-C. J Neurosci. 2001;21(18):7215-7225.

39. Laywell ED, Steindler DA. Boundaries and wounds, glia and glycoconjugates. Cellular and molecular analyses of developmental partitions and adult brain lesions. Ann NY Acad Sci. 1991;633:122-141.

40. GrandPre T, Nakamura F, Vartanian T, et al. Identification of the Nogo inhibitor of axon regeneration as a Reticulon protein. Nature. 2000;403(6768):439-444.

41. Yang J, Yu L, Bi AD, et al. Assignment of the human reticulon 4 gene (RTN4) to chromosome 2 p14 $\rightarrow 2$ p13 by radiation hybrid mapping. Cytogenet Cell Genet. 2000;88(1-2):101-102.

42. Karnezis T, Mandemakers W, McQualter JL, et al. The neurite outgrowth inhibitor Nogo A is involved in autoimmune-mediated demyelination. Nature Neurosci. 2004;7(7):736-744.

43. Trapp BD. Myelin-associated glycoprotein. Location and potential functions. Ann N Y Acad Sci. 1990;605:29-43.

44. Arquint M, Roder J, Chia LS, et al. Molecular cloning and primary structure of myelin-associated glycoprotein. Proc Natl Acad Sci U S A 1987;84(2):600-604.

45. Bartsch S, Montag D, Schachner M, et al. Increased number of unmyelinated axons in optic nerves of adult mice deficient in the myelinassociated glycoprotein (MAG). Brain Res. 1997;762(1-2):231-234.

46. Edgar JM, Garbern J. The myelinated axon is dependent on the myelinating cell for support and maintenance: molecules involved. $J$ Neurosci Res. 2004;76(5):593-598.

47. Sandvig A, Berry M, Barrett LB, et al. Myelin-, reactive glia-, and scarderived CNS axon growth inhibitors: Expression, receptor signaling, and correlation with axon regeneration. Glia. 2004;46(3):225-251.

48. Poliak S, Peles E. The local differentiation of myelinated axons at nodes of Ranvier. Nat Rev Neurosci. 2003;4:968-980. 
49. Tamagnone L, Artigiani S, Chen $\mathrm{H}$, et al. Plexins are a large family of receptors for transmembrane, secreted, and GPI-anchored semaphorins in vertebrates. Cell. 1999;99(1):71-80.

50. SEMA4D sema domain, immunoglobulin domain (Ig), transmembrane domain (TM) and short cytoplasmic domain, (semaphorin) 4D. Gene. 2017.

51. Ito Y, Oinuma I, Katoh $\mathrm{H}$, et al. Sema4D/plexin-B1 activates GSK-3 $\beta$ through R-Ras GAP activity, inducing growth cone collapse. EMBO Rep. 2006;7(7):704-709.

52. Kumanogoh A, Watanabe C, Lee I, et al. Identification of CD72 as a lymphocyte receptor for the class IV semaphorin CD100: a novel mechanism for regulating B cell signaling. Immunity. 2000;13(5):621631.

53. Ishida I, Kumanogoh A, Suzuki K, et al. Involvement of CD100, a lymphocyte semaphorin, in the activation of the human immune system via CD72: implications for the regulation of immune and inflammatory responses. Int Immunol. 2003;15(8):1027-1034.

54. Deborah WA, Megumi W, Olivia G, et al. The CD100 Receptor Interacts with Its Plexin B2 Ligand to Regulate Epidermal $\gamma \delta$ T Cell Function. Immunity. 2012;37(2):314-325.

55. Shiho F, Kenta M, Marcia T, et al. Neurotrophic effect of Semaphorin 4D in PC12 cells. Biochem Biophys Res Commun. 2003;301(2):304-310.

56. Struzyna LA, Katiyar K, Cullen DK. Living scaffolds for neuroregeneration. Curr Opin Solid ST M. 2014;18(6):308-318.

57. Lindvall O, Kokaia Z. Stem cells in human neurodegenerative disorderstime for clinical translation. J Clin Invest. 2010; 120(1): 29-40.

58. Pittenger MF, Mackay AM, Beck SC, et al. Multilineage potential of adult human mesenchymal stem cells. Science. 1999;284(5411):143147.

59. Makino S, Fukuda K, Miyoshi S, et al. Cardiomyocytes can be generated from marrow stromal cells in vitro. J Clin Invest. 1999;103(5):697-705.

60. Woodbury D, Schwarz EJ, Prockop DJ, et al. Adult rat and human bone marrow stromal cells differentiate into neurons. $J$ Neurosci Res. 2000;61(4):364-370.

61. Sanchez-Ramos J, Song S, Cardozo-Pelaez F, et al. Adult bone marrow stromal cells differentiate into neural cells in vitro. Exp Neurol. 2000;164(2):247-256.
62. Hermann A, Maisel M, Storch A. Epigenetic conversion of human adult bone mesodermal stromal cells into neuroectodermal cell types for replacement therapy of neurodegenerative disorders. Expert Opin Biol Ther. 2006;6(7):653-670.

63. Chen J, Chopp M. Neurorestorative treatment of stroke: cell and pharmacological approaches. NeuroRx. 2000;3(4):466-473.

64. Paul G, Anisimov SV. The secretome of mesenchymal stem cells: Potential implications for neuroregeneration. Biochimie. 2013;95(12): 2246-2256.

65. Place ES, Evans ND, Stevens MM. Complexity in biomaterials for tissue engineering. Nat Mater. 2009;8:457-470.

66. Dvir T, Timko BP, Kohane DS, et al. Nanotechnological strategies for engineering complex tissues. Nat Nanotechnol. 2011;6(1): 13-22.

67. Krishnan A, Dujardin E, Ebbesen TW, et al. Young's modulus of singlewalled nanotubes. Phys Rev B. 1998;58(20): 14013-14019.

68. Gilmore JL, Yi X, Quan L, Kabanov AV. Novel nanomaterials for clinical neuroscience. J Neuroimmune Pharmacol. 2008;3(2):83-94.

69. Kotov NA, Winter JO, Clements IP, et al. Nanomaterials for neural interfaces. Advanced Materials. 2009;21(40):3970-4004.

70. Lavdas AA, Papastefanaki F, Thomaidou D, et al. Cell adhesion molecules in gene and cell therapy approaches for nervous system repair. Curr Gene Ther. 2011;11(2):90-100.

71. Geiger B, Spatz JP, Bershadsky AD. Environmental sensing through focal adhesions. Nat Rev Mol Cell Biol. 2009;10(1): 21-33.

72. Skaper SD. Neuronal growth-promoting and inhibitory cues in neuroprotection and neuroregeneration. Methods Mol Biol. 2012;846:13-22.

73. Ni Y, Hu H, Malarkey EB, et al. Chemically functionalized water soluble single-walled carbon nanotubes modulate neurite outgrowth. J Nanosci Nanotechnol. 2005;5(10):707-712.

74. Malarkey EB, Reyes RC, Zhao B, et al. Water soluble single-walled carbon nanotubes inhibit stimulated endocytosis in neurons. Nano Lett. 2008;8(10):3538-3542.

75. Roman JA, Niedzielko TL, Haddon RC, et al. Single-walled carbon nanotubes chemically functionalized with polyethylene glycol promote tissue repair in a rat model of spinal cord injury. $J$ Neurotrauma. 2011;28(11):2349-2362. 